\title{
Axitinib for the Treatment of Metastatic Renal Cell Carcinoma
}

\author{
Recommendations for Therapy Management \\ to Optimize Outcomes
}

\author{
James Larkin, MD, PhD,* Mayer Fishman, MD, PhD, $\dagger$ Laura Wood, MSN, $\neq$ Sylvie Negrier, MD,* \\ Kara Olivier, NP, §Linda Pyle, BSc, * Vera Gorbunova, MD, \| Eric Jonasch, MD, 9 Lori Andrews, RN,\# \\ and Michael Staehler, MD**
}

\begin{abstract}
Axitinib is a novel, oral, multitargeted tyrosine kinase inhibitor, which inhibits vascular endothelial growth factor receptors 1 , 2 , and 3 at subnanomolar concentrations in vitro. In the phase III clinical trial in patients with metastatic renal cell carcinoma, axitinib showed a high objective response rate, and significantly prolonged progression-free survival compared with sorafenib. Thus, it is the first drug that has proven the concept of sequencing tyrosine kinase inhibitors in second-line treatment in a phase III prospective randomized trial. Although generally well tolerated and associated
\end{abstract}

From the *Renal Cancer Unit, Department of Medicine, Royal Marsden, London, UK; $†$ H. Lee Moffitt Cancer Center, Tampa, FL; \$Cleveland Clinic Cancer Institute, Cleveland, OH; §Massachusetts General Hospital, Boston, MA; \|N.N. Blokhin Russian Cancer Research Center, Moscow, Russia; M.D. Anderson Cancer Center, Houston; \#Texas Oncology PA Sammons Cancer Center/Baylor, Dallas, TX; and **Department of Urology, University of Munich, Munich, Germany.

J.L. is currently receiving consulting fees from Pfizer, Novartis, and GSK; grants (to his institution) from Pfizer and Novartis; and compensation for speaker's bureau services from Pfizer and Novartis. M.F. is currently receiving consulting fees from Bayer/Onyx, Eisai, Novartis, Pfizer, and Prometheus, and compensation for speaker's bureau services from Pfizer, Bayer, Prometheus, Novartis, and GSK; has received honoraria from Pfizer; compensation for speaker's bureau services from Genentech, educational presentations from Novartis and Wyeth; and travel support from Pfizer and compensation from Novartis and Prometheus; and his institution is currently receiving a research funding from Altor, Amgen, Aveo, Bayer/Onyx, Eisai, Novartis, Pfizer, and Prometheus. L.W. is on the speaker's bureau and has received travel support and honoraria from Pfizer. S.N. has received honoraria and travel support from Pfizer. K.O. has received has received honoraria and travel support from Pfizer. L.P. has received research funding, compensation for speaker's bureau services, educational presentations, and honoraria from Pfizer and compensation from Novartis and GSK. V.G. has received honoraria and travel support from Pfizer. E.J. receives consulting fees from Aveo, BMS, GSK, Novartis, and research funding Pfizer and has received honoraria and travel support from Pfizer. His institution receives research funding from Aveo, GSK, Novartis, and Pfizer. L.A. is currently receiving consulting fees and compensation for speaker's bureau services from Bayer; has received honoraria and travel support from Pfizer, and is employed by US Oncology. M.S. is a board member, is currently receiving consultancy fees, research funding, compensation for speaker's bureau services; receives travel support; and received compensation for expert testimony, educational presentations, honoraria, and travel support from Pfizer. Pfizer sponsored a meeting held to discuss data, after which a paper was written based on the meeting results. All authors were paid honoraria and M.F., L.W., S.N., K.O., V.G., E.J., L.A., and M.S. received travel support for the meeting itself (section 2 disclosures), but not for the paper development (section 1 disclosure).

Reprints: James Larkin, MD, PhD, Renal Cancer Unit, Department of Medicine, Royal Marsden Hospital, London SW3 6JJ, UK. E-mail: james.larkin@rmh.nhs.uk.

Copyright (C) 2013 by Lippincott Williams \& Wilkins

ISSN: 0277-3732/14/3704-0397

DOI: $10.1097 /$ COC.0b013e31827b45f9 with a low incidence of grade 3 or 4 toxicities, axitinib shows a distinct pattern of adverse events that require monitoring and management. The most common adverse events observed with axitinib include diarrhea, hypertension, fatigue, nausea, and vomiting. This article summarizes the most important adverse events observed and proposes recommendations for their monitoring, prevention, and treatment. The recommendations are based on the existing literature and discussion by an expert group of international physicians and nurses specialized in oncologic treatment of metastatic renal cell carcinoma, which gathered in July 2011 in London, UK. Proactive assessment and management of adverse events during axitinib therapy can minimize treatment interruptions and ensure optimal effect of treatment.

Key Words: axitinib, toxicity, side effect management, treatment optimization, renal cell carcinoma, tyrosine kinase inhibitor

(Am J Clin Oncol 2014;37:397-403)

Targeted therapies have substantially improved outcomes for patients with metastatic renal cell carcinoma (mRCC) in recent years. ${ }^{1}$ Several targeted therapies are now licensed for the treatment of mRCC, such as the monoclonal antibody bevacizumab; tyrosine kinase inhibitors (TKIs) sunitinib, sorafenib, pazopanib, and axitinib; and the mammalian target of rapamycin inhibitors temsirolimus and everolimus. ${ }^{2,3}$ Among these, the most recent addition to the mRCC treatment guidelines is axitinib, an oral, multitargeted TKI, which inhibits vascular endothelial growth factor (VEGF) receptors 1 , 2 , and 3 at subnanomolar concentrations in vitro. ${ }^{4}$ Agents in development such as tivozanib, dovitinib, and lenvatinib will not be discussed in this paper.

The importance of optimal proactive therapy management (management of adverse events [AEs]) in patients receiving targeted therapies for the treatment of $\mathrm{mRCC}$ is now well recognized, and helps to minimize treatment interruptions and ensure maximal outcomes from treatment. ${ }^{5}$ Although the general profile of AEs with axitinib is similar to that observed with other targeted therapies, there are also some key differences, particularly with respect to severity and frequency of occurrence. This may partly be influenced by the timing of treatment as axitinib is used in the second-line setting. As such, patients may be impacted by factors such as prior exposure to the AEs associated with other targeted agents, a higher disease burden, and lower performance status than patients receiving first-line therapy.

Axitinib was evaluated in 2 phase II studies in mRCC patients with either prior cytokine therapy or prior sorafenib therapy and demonstrated clinical activity in both studies. ${ }^{6,7}$ 
Subsequently, a phase III study compared axitinib with sorafenib for the treatment of mRCC patients who had progressed after exactly 1 prior treatment with sunitinib, bevacizumab + interferon- $\alpha$, temsirolimus, or cytokine-based therapy (the AXIS study). ${ }^{8}$

Axitinib was associated with significantly longer progression-free survival (PFS), the primary endpoint, compared with sorafenib (6.7 vs. $4.7 \mathrm{mo}$, respectively; hazard ratio [HR], 0.665; 95\% confidence interval [CI], 0.544-0.812; $P<0.0001){ }^{8}$ The subset of patients treated previously with cytokines achieved median PFS of 12.1 months with axitinib and 6.5 months with sorafenib (HR, 0.464; 95\% CI, 0.318$0.676 ; P<0.0001) .{ }^{8}$ In a subset of patients previously treated with sunitinib, median PFS for axitinib-treated and sorafenibtreated patients was 4.8 and 3.4 months, respectively (HR, $0.741 ; 95 \%$ CI, 0.573-0.958; $P=0.0107){ }^{8}$

In addition, the objective response rate (ORR) according to the Response Evaluation Criteria In Solid Tumors criteria v1.1, a secondary endpoint, was $19 \%$ for axitinib and $9 \%$ for sorafenib $(P=0.0001$; independent review committee assessment) ${ }^{8}$ In this study, patients in the axitinib group were twice as likely to have a favorable response to treatment compared with patients in the sorafenib group (risk ratio, 2.1; 95\% CI, 1.4-3.0; $P=0.0001) .{ }^{9}$ There were no statistically significant differences in median rates of overall survival (OS) between the axitinib and sorafenib arms in the overall study population $(\mathrm{HR}, 0.97)$ or in the prior sunitinib (HR, 1.00) and prior cytokine therapy (HR 0.81) subgroups (data on file, Pfizer Inc.). ${ }^{10}$

Preliminary data from a further phase III (INTORSECT) comparator study of second-line therapy with a targeted agent (either temsirolimus or sorafenib) after prior sunitinib for advanced RCC were recently reported (press release, May 15, 2012). The difference in PFS (the primary endpoint) was numerically higher in patients treated with temsirolimus, but not statistically significant. OS, a secondary endpoint, was significantly greater in the sorafenib arm. AEs were consistent with the known safety profiles of each single agent. In all 3 of the axitinib studies, axitinib demonstrated a manageable toxicity profile. Commonly observed AEs include gastrointestinal disturbances (diarrhea, nausea, and decreased appetite), hypertension, fatigue, and dysphonia. ${ }^{6-8}$ Proteinuria will also be discussed. Management of these AEs is based on experience in patients treated with axitinib and other TKI drugs used in kidney cancer therapy. For the clinician, optimal use of axitinib will include an awareness and organized plan to address these AEs as they occur for individual patients on treatment.

This article describes the AE profile of axitinib, and treatment recommendations developed by a global multidisciplinary panel regarding the management of specific AEs.

\section{METHODS}

A global multidisciplinary panel consisting of medical oncologists and oncology nurses met on July 29, 2011 in London, UK to review the safety and tolerability of axitinib at a meeting sponsored by the manufacturer, Pfizer Inc. At the meeting, each topic was introduced by presentation of available axitinib data from the phase III clinical study, provided by Pfizer Inc. The objectives of the meeting were to discuss the management of selected common axitinib AEs, to consider existing treatment algorithms and, where feasible, to develop consensus treatment recommendations for their clinical management. Incidence rates, $\mathrm{AE}$ onset and duration, and frequency of dose adjustments were discussed. In the clinical data presented, AEs were graded according to the National Cancer Institute Common Toxicity Criteria for Adverse Events (NCI CTCAE) version 3. ${ }^{11}$

\section{RESULTS}

\section{Axitinib Dosing and Titration}

In the phase III trial, the recommended starting dose for axitinib is $5 \mathrm{mg}$ twice daily (bid). For patients who are able to tolerate the initial dose of $5 \mathrm{mg}$ bid with no AEs $\geq$ grade 2 for 2 consecutive weeks, who are, according to CTCAE version 3 , normotensive (blood pressure [BP] $<150 / 90 \mathrm{~mm} \mathrm{Hg}$ ), and not receiving antihypertensive medication, initial dose adjustments to $7 \mathrm{mg}$ bid followed by $10 \mathrm{mg}$ bid may be possible. Conversely, dose reduction is recommended in patients who develop $\geq$ grade 3 nonhematologic toxicity. The dose may be reduced to $3 \mathrm{mg}$ bid, initially, or to $2 \mathrm{mg}$ bid, when more persistent or severe AEs occur, until the AEs improve to a severity $\leq$ grade 2 . Treatment discontinuation is generally recommended when grade 4 hematologic or nonhematologic AEs occur; however, axitinib treatment may be resumed at a lower dose level once the $\mathrm{AE}$ improves to a severity $\leq$ grade 2 .

\section{Diarrhea}

\section{Incidence and Onset}

Diarrhea was the most common AE in both arms of the axitinib phase III study (axitinib: all grade $55 \%$ of patients, $\geq$ grade $3,11 \%$; sorafenib: all grade $53 \%$, $\geq$ grade $3,7 \%$ ), indicating that the incidence of diarrhea with axitinib treatment is similar to that observed with other TKIs. In the phase II study among cytokine-pretreated patients, an increase in diarrhea severity was associated with response to treatment, ${ }^{7}$ suggesting that axitinib treatment should be continued in patients who are responding while instituting effective diarrhea management.

The onset of diarrhea is not acute in patients receiving axitinib, usually developing during weeks 3 to 8 of treatment. The development of diarrhea is generally unpredictable and shows substantial inter-patient variability, rendering risk stratification and generalized prophylaxis inappropriate. Clinical experience suggests that the development of therapyinduced diarrhea with previous regimens does not predict axitinib-induced diarrhea, ${ }^{12}$ however, poor performance status is a risk factor for diarrhea development. ${ }^{12}$

\section{Mechanism and Characteristics}

At present, the mechanism underlying the occurrence of diarrhea in patients treated with VEGF-targeted agents remains unclear, but may involve direct damage to the intestinal mucosa. ${ }^{13,14}$ Other potential explanations are that these agents cause changes to the normal intestinal microflora or induce dysmotility through effects on the Cajal cells responsible for colonic motor function. ${ }^{13}$ Diarrhea may be worsened or caused by factors including diet, infection, other types of bowel pathology, concomitant medications, neuroendocrine factors (eg, hyperthyroidism and diabetes), and anxiety, highlighting the need for careful patient assessment. ${ }^{12}$ Diarrhea is a frequent cause of dose reductions or interruptions and requires proactive management.

\section{Management}

In general, management of diarrhea relies on patient education, dietary modification, use of antidiarrheal medication, and recourse to dose reduction and intravenous 
hydration if required (Table 1). The consistency and volume of stool and duration of diarrhea, presence of fever, or signs and symptoms of dehydration should all be assessed. ${ }^{12}$ A careful medical and medication history may identify underlying causative or exacerbating factors. ${ }^{12}$

Diarrhea can usually be managed by dietary modification and oral hydration/electrolyte replacement and through the use of antidiarrheal medications, such as loperamide on an asneeded basis. ${ }^{15,16}$ However, patients should not overuse antidiarrheals, as they can cause constipation, especially in patients taking opioids, and this can be even more troublesome for the patient than diarrhea. Probiotics and psyllium products may also be useful. ${ }^{15,17,18}$ Proactive drug breaks of 1 to 2 days may help to relieve diarrhea and help the patient's psychological well-being. Dose reduction and outpatient intravenous hydration are recommended for grade 3 diarrhea. Patients with grade 4 diarrhea (defined as life threatening) should stop taking axitinib until the diarrhea resolves to $\geq$ grade 2 , when axitinib can be reintroduced at 1 dose level lower. Patients with diarrhea of this severity may also need to be hospitalized for intensive hydration and bowel rest.

\section{Nausea, Vomiting, and Decreased Appetite Incidence and Onset}

Nausea and vomiting are frequently observed with many anticancer regimens, and may impact patient compliance and persistence on therapy. In general, the incidence of nausea and vomiting with TKIs can be up to $30 \%,{ }^{5}$ resembling the results in the AXIS study. Thirty-two percent of axitinib-treated patients experienced nausea and $24 \%$ reported vomiting ( $3 \%$ of patients experienced $\geq$ grade 3$){ }^{8}$ These results indicate that axitinib has low emetogenic potential.

\section{Mechanism and Characteristics}

The exact mechanism by which VEGF receptor inhibitors cause nausea and vomiting is unclear; however, similar mechanisms to those of chemotherapeutic agents may be responsible. Antineoplastic agents stimulate enteroendocrine cells in the proximal small intestine, leading to the release of local mediators, including 5-hydroxytryptamine (5-HT; serotonin), substance $\mathrm{P}$, and cholecystokinin. ${ }^{19}$ These mediators bind to adjacent vagal afferent fibers, which project to the dorsal brain stem initiating the central vomiting reflex. ${ }^{19}$ In clinical practice, nausea, vomiting, dyspepsia, and reduced appetite often occur together and may exacerbate one another, although the link between these events is not always straightforward. Effective prevention or management of acute nausea and vomiting is critical to minimize the impact on subsequent treatment.

\section{Management}

Small, frequent meals and avoiding spicy foods may help alleviate symptoms; some patients may also benefit from consultation with a nutritionist. Assessment of any baseline conditions, which may exacerbate nausea and vomiting, is advised, including dyspepsia, anorexia, and early satiety. Administration of a proton pump inhibitor or $\mathrm{H}_{2}$ antagonist may be useful to prevent dyspepsia, which can mimic nausea. ${ }^{20}$ Patients should be advised to avoid taking a proton pump inhibitor or $\mathrm{H}_{2}$ antagonist at the same time as axitinib and to seek medical attention if they develop abdominal pain.

Choice of antiemetic therapy should be guided by the patient's preference, medical history, and concomitant medications, keeping in mind the potential adverse effects of the antiemetics, such as constipation with 5-HT3 receptor antagonists. ${ }^{21}$ After baseline assessments, patients should also be reassessed for any changes to their physical status (especially weight), appetite, comorbidities, and medications. No change to the axitinib dose is indicated for grade 1 or 2 nausea or vomiting, but patients should be counseled to maintain fluid intake; some may also benefit from dietary modifications and initiation of antiemetic therapy. Proactive intravenous hydration is indicated in patients with grade 2 vomiting and dose reduction may be necessary for grade 3 nausea or vomiting. Treatment should be temporarily discontinued for grade 4 nausea or vomiting, and restarted at 1 dose level lower after symptoms improve to $\leq$ grade 2 . Active hydration and replacement of electrolytes may be particularly important for grades 3 or 4 nausea and vomiting, and hospitalization may be indicated.

\section{Hypertension}

\section{Incidence and Onset}

Hypertension of any grade occurred in $40 \%$ of patients treated with axitinib in the phase III AXIS study, with $\geq$ grade 3 hypertension occurring in $16 \%$ of patients. ${ }^{8}$ The onset of hypertension during axitinib therapy is rapid, with an increase in diastolic blood pressure (dBP) of approximately $10 \mathrm{~mm} \mathrm{Hg}$ apparent by day 4 of treatment. ${ }^{22}$

\section{Mechanism}

BP elevation is a common phenomenon with VEGF inhibitors and an increase in $\mathrm{dBP}$ may be a marker for the pharmacodynamic effect of these agents. ${ }^{23,24}$ Although the precise mechanism of the hypertensive effect of VEGF inhibitors has not been elucidated, it may involve inhibition of the endothelial nitric oxide (NO) synthase pathway, the signaling of which is mediated by the VEGF receptor. ${ }^{23} \mathrm{As} \mathrm{NO}$ is an endothelium-dependent vasodilator, inhibition of $\mathrm{NO}$

\section{TABLE 1. Recommendations for the Management of Diarrhea}

\section{Baseline assessments and patient Assess baseline bowel habits} education

Review concomitant medications

Educate patients about the likelihood diarrhea may develop

Products

Probiotics may reduce frequency and severity

Grade 1 or 2

Treatment with bulking agents or pancreatic enzyme therapy may also be beneficial

Grade 3 or $4 \quad$ Orade 3 , decrease dose by 1 dose leve
improvement to CTCAE $\leq$ grade 2

Dietary modification

Oral hydration and antidiarrheal medications: loperamide or diphenoxylate

Outpatient intravenous hydration

Consider hospital admission for hydration and bowel rest for grade 4 
production may increase vascular tone and peripheral resistance. ${ }^{14,23}$

\section{Management}

Before initiating axitinib therapy, patients should be educated regarding the potential occurrence of hypertension and the need to monitor BP during treatment. It is important to regularly monitor BP during the first week of therapy and the panel recommends assessments at baseline and, at a minimum, on days 3 to 6 , and regularly thereafter. In addition, using 5 to 6 serial measurements over several days ensures an accurate assessment of BP, as BP may fluctuate from day-to-day. ${ }^{25}$

Increase in $\mathrm{dBP}$ may be an indication of axitinib efficacy; results from a retrospective analysis across 5 phase II studies in 4 different tumor types indicated that axitinib efficacy correlated with $\mathrm{dBP} \geq 90 \mathrm{~mm} \mathrm{Hg} .{ }^{24}$ In this analysis, patients with $\mathrm{dBP} \geq 90 \mathrm{~mm} \mathrm{Hg}$ had a significantly lower relative risk of death than those with $\mathrm{dBP}<90 \mathrm{~mm} \mathrm{Hg}$ (adjusted HR, 0.55; 95\% CI, $0.39-0.77 ; P<0.001) .{ }^{24}$ The relative risk of progression was also lower in patients with $\mathrm{dBP} \geq 90 \mathrm{~mm} \mathrm{Hg}$ (HR, 0.76 ; 95\% CI, 0.54-1.06; $P=0.107$ ), and ORR was significantly higher $(43.9 \%$ vs. $12.0 \% ; P<0.001) .{ }^{24}$ In addition, an 8-week landmark analysis demonstrated higher median OS (25.8 vs. $14.9 \mathrm{mo})$ and higher median PFS (10.2 vs. $7.1 \mathrm{mo})$ for patients with $\mathrm{dBP} \geq 90 \mathrm{~mm} \mathrm{Hg} .{ }^{24}$ A prospective randomized phase II double-blind study (NCT00835978) examining the correlation between BP and efficacy with axitinib has completed accrual and preliminary data indicate that patients $(\mathrm{n}=18)$ with mean increases in $\mathrm{dBP} \geq 15 \mathrm{~mm} \mathrm{Hg}$ on day 15 of cycle 1 achieved a higher ORR than those $(n=36)$ with a mean change in $\mathrm{dBP} \leq 15 \mathrm{~mm} \mathrm{Hg}\left(61 \%\right.$ vs. $53 \%$, respectively). ${ }^{26}$ Final data are awaited.

BP elevations to hypertensive levels, currently defined as $>140 / 90 \mathrm{~mm} \mathrm{Hg}$ should be treated with antihypertensive therapy. This should be combined with appropriate monitoring for response to the antihypertensive agent selected for treatment. Table 2 lists the panel's recommendations for use of antihypertensive drugs with axitinib based on existing guidelines. Angiotensin converting enzyme (ACE) inhibitors and dihydropyridine calcium channel blockers are preferred for the treatment of hypertension, followed by diuretics and $\beta$-blockers. Nondihydropyridine calcium channel blockers should be used cautiously with CYP3A4 inhibitors, such as axitinib. In addition to antihypertensive therapy, patients should be advised to make healthy lifestyle choices, including regular exercise, weight control, and moderate alcohol consumption. This should be combined with counseling on how to recognize signs of potentially dangerous and emergent BP elevations, such as flushing, headache, and a pounding heart.

Downward axitinib dose adjustments may be required in some instances where antihypertensive therapy fails to control $\mathrm{BP}$ to recommended levels. Axitinib therapy may need to be interrupted for severe persistent hypertension, but can be restarted at the next lower dose once the patient's BP has normalized. Patients receiving antihypertensive medication should be monitored for the occurrence of hypotension, which may occur when axitinib is discontinued.

\section{Fatigue \\ Incidence and Onset}

Fatigue is a common symptom in patients with cancer. The incidence of fatigue varies greatly among TKI treatments in patients with mRCC (19\% to $73 \%$ and $3 \%$ to $11 \%)$; all grade and grade $3 / 4$, respectively. ${ }^{27}$ Fatigue, marked with persistent and subjective exhaustion or tiredness inconsistent with recent activity, can have a significant impact on quality of life.

Both physical and mental factors may contribute to fatigue and distinguishing between the 2 remains challenging. In the phase III AXIS study, $\geq$ grade 3 fatigue was reported in $11 \%$ of patients. ${ }^{8}$ In the panel's clinical experience, onset of fatigue was associated with dose escalations. Occurrence was observed between the third week of the initial treatment cycle and up to the third cycle, but was most common at week 3 or 4 . The level of fatigue appeared to plateau for some patients depending on their baseline levels. Importantly, fatigue during axitinib treatment was often reversible a few days after a temporary treatment hiatus. The panel considered dose interruptions more suitable for managing fatigue than dose reductions in patients receiving axitinib treatment.

\section{Mechanism and Characteristics}

The underlying mechanism of fatigue in cancer patients is undoubtedly multifactorial, but may involve circadian rhythm disruption, alterations in muscle and ATP metabolism, genetic factors, and dysregulation of cytokines, the hypothalamicpituitary-adrenal axis, serotonin, or vagal afferent nerves. ${ }^{28}$ In patients treated with axitinib, fatigue may be related to reduction in thyroid hormone levels, as the time course of thyroid stimulating hormone increases correlates with fatigue occurrence. ${ }^{29}$ Fatigue may also be caused or exacerbated by underlying factors (eg, nutritional deficits and deconditioning), symptoms (eg, pain), medication side effects (eg, sedation), anemia, emotional distress (eg, depression and anxiety), and comorbidities (eg, endocrine, cardiac, renal, hepatic or

\begin{tabular}{|c|c|c|}
\hline $\begin{array}{l}\text { Preference/ } \\
\text { Order }\end{array}$ & Class & Examples \\
\hline \multirow[t]{3}{*}{ First } & $\begin{array}{l}\text { Angiotensin converting } \\
\text { enzyme inhibitors }\end{array}$ & $\begin{array}{l}\text { Benazepril, captopril, enalapril, fosinopril, imidapril, lisinopril, quinapril, perindopril, ramipril } \\
\text { zofenopril }\end{array}$ \\
\hline & Angiotensin receptor blockers & Candesartan cilexetil, eprosartan, irbesartan, olmesartan medoxomil, telmisartan, valsartan \\
\hline & $\begin{array}{l}\text { Dihydropyridine calcium } \\
\text { channel blockers }\end{array}$ & $\begin{array}{l}\text { Amlodipine, benidipine, cilnidipine, felodipine, isradipine, lacidipine, manidipine, nicardipine, } \\
\text { nifedipine, nilvadipine, nisoldipine, nitrendipine }\end{array}$ \\
\hline \multirow{3}{*}{ Second } & Potassium-sparing diuretics & Amiloride, spironolactone, triamterene \\
\hline & Loop diuretics & Bumetanide, furosemide \\
\hline & Thiazide diuretics & Chlorothiazide, chlorthalidone, hydrochlorothiazide, indapamide \\
\hline $\begin{array}{l}\text { Use with } \\
\text { caution }\end{array}$ & $\beta$-blockers & Atenolol, metoprolol, propranolol, nadolol, nebivolol, propranolol \\
\hline Avoid & $\begin{array}{l}\text { Nondihydropyridine calcium } \\
\text { channel blockers }\end{array}$ & Diltiazem, verapamil \\
\hline
\end{tabular}




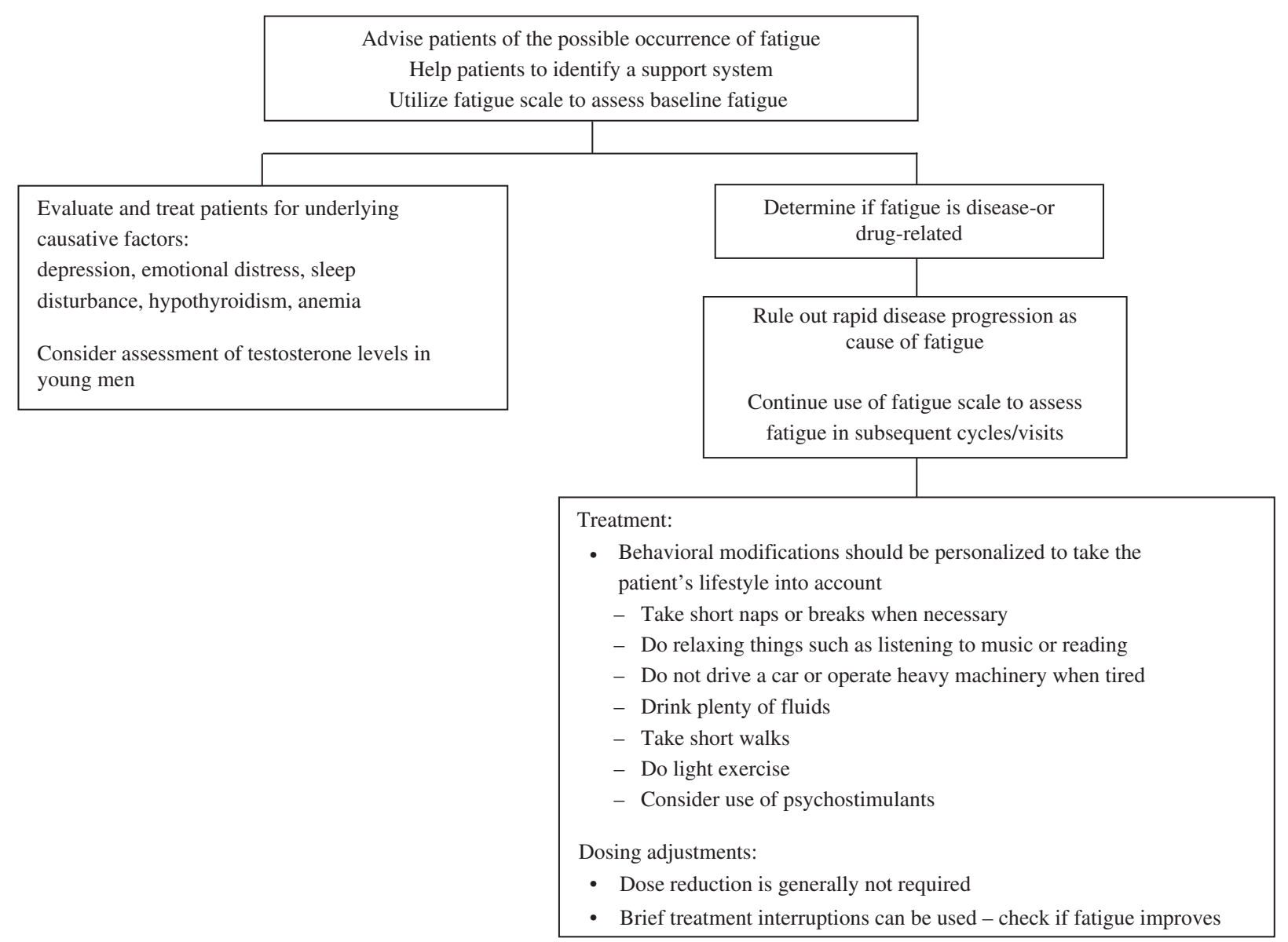

FIGURE 1. Suggested algorithm for the management of axitinib-related fatigue. [Republished with permission from: Kollmannsberger C, Soulieres D, Wong R, et al. Sunitinib therapy for metastatic renal cell carcinoma: recommendations for management of side effects. Can Urol Assoc J. 2007;1:S41-S45. Copyright 2011]. ${ }^{30}$

pulmonary dysfunction, or infection). The multifaceted etiology of fatigue highlights the need for careful assessment.

\section{Management}

There are several practical steps that may be taken to minimize impact and manage fatigue. The general principles detailed by Kollmannsberger et $\mathrm{al}^{30}$ for managing fatigue are also applicable to managing fatigue in patients treated with axitinib (Fig. 1). The panel also considered the utilization of guidelines such as those of the NCCN useful. ${ }^{28}$ Before starting treatment, patients should be advised about the potential for fatigue development and educated on how to manage fatigue. The level of baseline fatigue and caregiver support should be assessed, as well as hemoglobin levels and nutritional status.

A focused assessment of patient history; use of prescription and over-the-counter medications and supplements; evaluation of onset, pattern, duration, and impact of fatigue; and social support and availability of caregivers is required to evaluate and treat fatigue. ${ }^{28}$ It is important that treatable contributing factors are identified and managed. Some concomitant medications such as $\mathrm{H}_{2}$ receptor antagonists and $\beta$ blockers can cause fatigue. Because of the subjective nature of fatigue, it is sometimes difficult to distinguish fatigue from other conditions, particularly affective disorders, such as depression. Psychostimulants such as methylphenidate or modafinil can be considered for patients with moderate or severe fatigue. ${ }^{31}$ Correction of thyroid hormone levels should be considered in all patients suffering from fatigue. In men, fatigue may be related to low levels of testosterone or dehydroepiandrosterone sulfate, and may benefit from testosterone substitution through injection, gel, or patch. In addition, patients with fatigue may benefit from general strategies to conserve energy, such as pacing themselves during activity and scheduling activities to times of peak energy. Patients experiencing fatigue should be encouraged to engage in a moderate level of physical activity or exercise and should consider energy-conserving strategies such as scheduling activities at times of peak energy and pacing themselves during activity. Moderate-intensity resistance training seems to have the greatest positive impact on fatigue. ${ }^{32}$ However, it is important to tailor the exercise program to the patient's age, sex, and general physical fitness, as well as the presence of comorbidities or underlying conditions such as anemia.

\section{Dysphonia}

\section{Incidence and Onset}

Dysphonia occurred in $31 \%$ of patients in the axitinib phase III study, but severity was limited to grade 1 or $2 .{ }^{8}$ Onset was observed during the first 2 weeks of treatment but resolved rapidly with a temporary treatment interruption ( 1 to $2 \mathrm{~d}$ ). 


\section{Mechanism and Characteristics}

Dysphonia, characterized by a harsh or rasping voice arising from or spreading to the larynx, manifests as changes in vocal quality, pitch, loudness, or vocal effort, and can impair communication and voice-related quality of life in affected patients. ${ }^{33}$ Dysphonia has been observed more commonly with newer VEGF inhibitors, such as tivozanib, ${ }^{34}$ than with firstgeneration agents. The severity of dysphonia caused by axitinib and other targeted agents seems to be moderate. ${ }^{6,8,35}$ In cases of dysphonia reported with other VEGF inhibitors, the true vocal folds appear atrophic and ischemic, and blood vessels in the epiglottis and laryngeal region appear abnormal, resembling telangiectasia. ${ }^{35}$ The mechanism of dysphonia induced by VEGF-targeted therapies remains unclear; however, the antiangiogenic effects of these agents may reduce vascularization or microvascular permeability in the mucosa or submucosa of the larynx, resulting in greater rigidity, a stiffer vocal fold and poor quality vocal vibrations, ${ }^{35-38}$ may cause asymmetry in the mucosal wave, or may have direct atrophic effects on the mucosa. ${ }^{35}$

\section{Management}

Patients should be educated about the possibility of dysphonia when initiating treatment with axitinib. Additional measures include objectively listening to the voice of a patient with hoarseness during treatment, taking a targeted medical history, and reviewing current medications. ${ }^{33}$ Other medications implicated in dysphonia development include ACE inhibitors, antihistamines, diuretics, anticholinergics, inhaled steroids, bisphosphonates, and antipsychotics. ${ }^{33}$ In the panel's clinical experience, dysphonia resolved rapidly after a 1- to 2day treatment interruption; physical examination of the head and neck was recommended if hoarseness persisted thereafter. Ample water and avoidance of irritants, such as tobacco smoke, and limitation of voice strain may be helpful. No pharmacological intervention is indicated for mild-to-moderate dysphonia. Referral to an ear, nose, and throat specialist is indicated in patients with more severe dysphonia ( $\geq$ grade 3 ), when hoarseness does not resolve within 3 months of onset, or whenever a serious underlying cause for vocal changes of any severity is suspected. ${ }^{33}$

\section{Proteinuria}

\section{Incidence and onset}

Proteinuria is a less common AE associated with axitinib treatment, occurring in $10 \%$ of axitinib-treated patients in the phase III AXIS study (Pfizer Inc., data on file). Although grade 3 or 4 proteinuria is uncommon with any of the VEGF receptor inhibitors, vigilant monitoring is recommended because of the insidious onset of proteinuria and the impact it can have on patient well-being and their ability to undergo further courses of treatment. The onset of proteinuria may be more rapid with agents targeting VEGF (eg, bevacizumab) rather than the VEGF receptor. ${ }^{39}$

\section{Mechanism and Characteristics}

VEGF is an important mediator of normal renal function, and is expressed on glomerular podocytes. ${ }^{39}$ Studies suggest that VEGF inhibition reduces endothelial fenestrations in glomerular capillaries, disrupting the filtration barrier and leading to proteinuria. ${ }^{14,39}$ The pathogenic changes seen in mice with targeted deletion of VEGF or treated with VEGF inhibitors include loss of endothelial fenestrations in glomerular capillaries, endotheliosis, and podocyte depletion. ${ }^{39}$ In addition, proteinuria may result from altered renal hemodynamics secondary to NO depletion or from glomerular injury secondary to hypertension. ${ }^{39}$

\section{Management}

Before initiating treatment with axitinib, patients should be asked about previous renal disease, and undergo a baseline assessment of renal function and proteinuria. ${ }^{39}$ Urinalysis by dipstick should be conducted monthly; if significant proteinuria is present, patients should have a quantitative $(24 \mathrm{~h})$ protein assessment or be considered for referral to a specialist. Patients with proteinuria (1000 to $2000 \mathrm{mg} / 24 \mathrm{~h}$ ) can continue axitinib at the same dose level; ACE inhibitors or angiotensin II receptor blockers may be added to protect renal function if the patient's $\mathrm{BP}$ is $>130 / 80 \mathrm{~mm} \mathrm{Hg}{ }^{39}$ Potassium-sparing diuretics are preferred over thiazide, loop diuretics, and $\beta$-blockers, as the latter may contribute to fatigue. ${ }^{23}$ If proteinuria is $\geq 2 \mathrm{~g} / 24 \mathrm{~h}$, the axitinib dose should be reduced to the next lower level or temporarily discontinued and 24-hour urine collection repeated. After proteinuria falls below $2000 \mathrm{mg} / 24 \mathrm{~h}$, axitinib can be restarted at 1 dose level lower. Dietary changes (eg, protein and sodium restriction) are not recommended as patients are often already on a modified diet to manage other AEs.

\section{CONCLUSIONS}

Axitinib has proven efficacy in patients with advanced RCC. AEs are generally mild-to-moderate, manageable with standard medical interventions, and do not usually require treatment discontinuation. Recommendations for monitoring, preventing, and treating AEs can reduce side effect severity and duration, as well as improve dose delivery, which may maximize clinical outcomes. Strategies include educating patients about potential AEs, regular monitoring, and concomitant treatment of AEs. With the recommendations described here, the majority of AEs associated with axitinib can be managed while maintaining patients on therapy. The mechanisms underlying the occurrence of these AEs are not fully characterized. Data from prospective randomized trials with axitinib can optimize axitinib treatment outcomes by providing insight into underlying mechanisms and relationships between certain AEs, such as hypertension, and efficacy.

\section{ACKNOWLEDGMENTS}

The authors thank C. Choi, L. Cisar, S. Hariharan, G. Maurin, J. Ryan, and C. Taylor of Pfizer Inc. for their support during the meeting held on July 29, 2011 in London, UK. Editorial support was provided by Minal Kotecha at ACUMED (Tytherington, UK) and was funded by Pfizer Inc.

\section{REFERENCES}

1. Coppin C, Le L, Porzsolt F, et al. Targeted therapy for advanced renal cell carcinoma. Cochrane Database Syst Rev. 2008;CD006017.

2. Ljungberg B, Cowan N, Hanbury DC, et al. EAU guidelines on renal cell carcinoma: the 2010 update. Eur Urol. 2010;58: 398-406.

3. National Comprehensive Cancer Network. NCCN Clinical Practice Guidelines in Oncology: Kidney Cancer v.2.2012. Available at: http://www.nccn.org/professionals/physician_gls/f_guidelines. asp. Accessed April 10, 2012.

4. Escudier B, Gore M. Axitinib for the management of metastatic renal cell carcinoma. Drugs R D. 2011;11:113-126.

5. Ravaud A. Treatment-associated adverse event management in the advanced renal cell carcinoma patient treated with targeted therapies. Oncologist. 2011;16(suppl. 2):32-44. 
6. Rini BI, Wilding G, Hudes G, et al. Phase II study of axitinib in sorafenib-refractory metastatic renal cell carcinoma. J Clin Oncol. 2009;27:4462-4468.

7. Rixe O, Bukowski RM, Michaelson MD, et al. Axitinib treatment in patients with cytokine-refractory metastatic renal-cell cancer: a phase II study. Lancet Oncol. 2007;8:975-984.

8. Rini BI, Escudier B, Tomczak P, et al. Comparative effectiveness of axitinib versus sorafenib in advanced renal cell carcinoma (AXIS): a randomised phase 3 trial. Lancet. 2011;378:1931-1939.

9. Rini BI, Escudier B, Tomczak P, et al. Axitinib versus sorafenib as second-line therapy for metastatic renal cell carcinoma (mRCC): Results of phase III AXIS trial. J Clin Oncol. 2011;29(suppl): Abstract 4503.

10. Michaelson MD, Rini BI, Escudier BJ, et al. Phase III AXIS trial of axitinib versus sorafenib in metastatic renal cell carcinoma: updated results among cytokine-treated patients. J Clin Oncol. 2012;30(suppl): Abstract 4546.

11. National Cancer Institute. Common Terminology Criteria for Adverse Events (CTCAE) v.3.0. 2006. Available at: http://ctep. cancer.gov/protocolDevelopment/electronic_applications/docs/ ctcaev3.pdf. Accessed November 20, 2012.

12. Richardson G, Dobish R. Chemotherapy induced diarrhea. J Oncol Pharm Pract. 2007;13:181-198.

13. Loriot Y, Perlemuter G, Malka D, et al. Drug insight: gastrointestinal and hepatic adverse effects of molecular-targeted agents in cancer therapy. Nat Clin Pract Oncol. 2008;5:268-278.

14. Kamba T, McDonald DM. Mechanisms of adverse effects of antiVEGF therapy for cancer. Br J Cancer. 2007;96:1788-1795.

15. Wood LS. Management of vascular endothelial growth factor and multikinase inhibitor side effects. Clin J Oncol Nurs. 2009; (13 suppl):13-18.

16. Kollmannsberger C, Soulieres D, Wong R, et al. Sunitinib therapy for metastatic renal cell carcinoma: recommendations for management of side effects. Can Urol Assoc J. 2007;1:S41-S54.

17. Wood LS. Managing the side effects of sorafenib and sunitinib. Commun Oncol. 2006;3:558-562.

18. Osterlund P, Ruotsalainen T, Korpela R, et al. Lactobacillus supplementation for diarrhoea related to chemotherapy of colorectal cancer: a randomised study. Br J Cancer. 2007;97:1028-1034.

19. Hesketh PJ. Chemotherapy-induced nausea and vomiting. $N$ Engl $J$ Med. 2008;358:2482-2494.

20. National Comprehensive Cancer Network. NCCN Clinical Practice Guidelines in Oncology: Antiemesis v.1.2012. Available at: http://www.nccn.org/professionals/physician_gls/PDF/antiemesis.pdf. Accessed April 10, 2012.

21. Kovac AL. Benefits and risks of newer treatments for chemotherapy-induced and postoperative nausea and vomiting. Drug Saf. 2003;26:227-259.

22. Fishman MN, Carducci M, Bair AH, et al. Axitinib pharmacokinetics and blood pressure changes in front-line metastatic renal cell carcinoma (RCC) patients. Ann Oncol. 2010;21(suppl. 8):284.

23. Maitland ML, Bakris GL, Black HR, et al. Initial assessment, surveillance, and management of blood pressure in patients receiving vascular endothelial growth factor signaling pathway inhibitors. J Natl Cancer Inst. 2010;102:596-604.
24. Rini BI, Schiller JH, Fruehauf JP, et al. Diastolic blood pressure as a biomarker of axitinib efficacy in solid tumors. Clin Cancer Res. 2011;17:3841-3849.

25. Powers BJ, Olsen MK, Smith VA, et al. Measuring blood pressure for decision making and quality reporting: where and how many measures? Ann Intern Med. 2011;154:781-788.

26. Rini BI, Grünwald V, Fishman MN, et al. Axitinib for first-line metastatic renal cell carcinoma (mRCC): Overall efficacy and pharmacokinetic (PK) analyses from a randomized phase II study. J Clin Oncol. 2012;30(suppl): Abstract 4503.

27. Larkin J, Pyle LM, Gore ME. Fatigue in renal cell carcinoma: the hidden burden of current targeted therapies. Oncologist. 2010; 15:1135-1146.

28. National Comprehensive Cancer Network. NCCN Clinical Practice Guidelines in Oncology: Cancer-Related Fatigue v.1.2011 Available at: http://www.nccn.org/professionals/physician_gls/ PDF/fatigue.pdf. Accessed April 10, 2012.

29. Mukohara T, Nakajima H, Mukai H, et al. Effect of axitinib (AG013736) on fatigue, thyroid-stimulating hormone, and biomarkers: a phase I study in Japanese patients. Cancer Sci. 2010;101:963-968.

30. Kollmannsberger C, Bjarnason G, Burnett P, et al. Sunitinib in metastatic renal cell carcinoma: recommendations for management of noncardiovascular toxicities. Oncologist. 2011;16: 543-553.

31. Breitbart W, Alici Y. Psychostimulants for cancer-related fatigue. $J$ Natl Compr Canc Netw. 2010;8:933-942.

32. Brown JC, Huedo-Medina TB, Pescatello LS, et al. Efficacy of exercise interventions in modulating cancer-related fatigue among adult cancer survivors: a meta-analysis. Cancer Epidemiol Biomarkers Prev. 2011;20:123-133.

33. Schwartz SR, Cohen SM, Dailey SH, et al. Clinical practice guideline: hoarseness (dysphonia). Otolaryngol Head Neck Surg. 2009;141:S1-S31.

34. De Luca A, Normanno N. Tivozanib, a pan-VEGFR tyrosine kinase inhibitor for the potential treatment of solid tumors. IDrugs. 2010;13:636-645.

35. Hartl DM, Ferte C, Loriot Y, et al. Dysphonia induced by vascular endothelium growth factor/vascular endothelium growth factor receptors. Invest New Drugs. 2010;28:884-886.

36. Baffert F, Thurston G, Rochon-Duck M, et al. Age-related changes in vascular endothelial growth factor dependency and angiopoietin-1-induced plasticity of adult blood vessels. Circ Res. 2004;94:984-992.

37. Baffert F, Le T, Sennino B, et al. Cellular changes in normal blood capillaries undergoing regression after inhibition of VEGF signaling. Am J Physiol Heart Circ Physiol. 2006;290: H547-H559.

38. Inai $\mathrm{T}$, Mancuso $\mathrm{M}$, Hashizume $\mathrm{H}$, et al. Inhibition of vascular endothelial growth factor (VEGF) signaling in cancer causes loss of endothelial fenestrations, regression of tumor vessels, and appearance of basement membrane ghosts. Am J Pathol. 2004; 165:35-52.

39. Izzedine $\mathrm{H}$, Massard $\mathrm{C}$, Spano JP, et al. VEGF signalling inhibition-induced proteinuria: Mechanisms, significance and management. Eur J Cancer. 2010;46:439-448. 